\title{
Linking Privacy Concerns for Traceable Information and Information Privacy Protective Responses on Electric Scooter Sharing Platforms
}

\author{
Lin $\mathrm{Li}$ \\ Kyung Hee University \\ lilin@khu.ac.kr \\ Younghoon Chang \\ Beijing Institute of Technology \\ younghoonchang@bit.edu.cn
}

\author{
Kyung Young Lee \\ Dalhousie University \\ kyunglee@dal.ca \\ Sung-Byung Yang \\ Kyung Hee University \\ sbyang@khu.ac.kr
}

\begin{abstract}
Electric scooter (e-scooter) sharing platforms have taken over multiple cities across the globe. Yet, behind the craze, information privacy issue has been added to the list of concerns in this revolution of e-scooter sharing, as major companies and even governments' regulatory bodies are alleged to collect and use traceable information generated by users' routes without proper notice. We therefore attempt to conceptualize a new dimension of privacy concern (i.e., privacy concerns for traceable information: PCTI) in the context of e-scooter sharing platforms. In an attempt to understand users' rising actions in protecting their privacy, we further examine the relationships among some antecedents, PCTI, and information privacy protective responses, drawing on the APCO macro model. Our research findings are expected to contribute to the body of knowledge on information privacy in the sharing economy context, and provide some practical implications to both users and industry members of e-scooter sharing services.
\end{abstract}

\section{Introduction}

The concept of the sharing economy refers to "the peer-to-peer-based activity of obtaining, giving, or sharing the access to goods and services, coordinated through community-based online services" (Hamari et al. 2016, p. 2047). Along with many sharing economy business models which have already been active such as Uber and Airbnb, the emerging electric scooter (escooter) sharing can be defined as the shared use of an e-scooter that enables riders to have short-term access on an on-demand basis, often serving as a last-mile public transit connections (Shaheen and Chan, 2016). After launched in the U.S. in the fall of 2017, e-scooter sharing has swept across the U.S. and Europe and has accounted for $45.8 \%$ (35 million trips out of 84 million trips taken) of all the shared mobility services in the U.S. in 2018 (Dickey, 2019). As a type of the newest trend, so-called Mobility-as-a-Service (MaaS), the concept of renting a means of transportation when needed with mobile apps has been raised to a new level with an advent of e-scooter sharing services (Gindrat, 2018). During the process, many people start to develop a kind of 'love/hate relationship' with these escooter sharing services. E-scooters are loved for their convenience, affordability, and eco-friendliness, while they are also hated for safety hazard and privacy concern. Safety hazard is obviously due to the fear of traffic accidents in most cases. But, recently, the debate of privacy concerns on e-scooter sharing services has been emerged in many metropolitan cities, such as San Francisco and Los Angeles (Nelson, 2019).

In academia as well, numerous aspects of privacy concerns have been studied in the field of e-commerce and information systems (IS) (Chang et al., 2018; Dinev and Hart, 2006; Smith et al., 2011). Especially, quite a few studies have discussed and investigated users' privacy concerns related to location-based services and their impact on users' attitudes and behaviors in diverse contexts of online/mobile services, such as marketing information provision services (Xu et al., 2009) and location-aware social network services (Sun et al., 2015). However, to the best of our knowledge, a detailed empirical examination on users' privacy concerns with their traceable location information and its impact on their behavior changes in the context of ride-sharing services remains underinvestigated, inspiring the motivation of this study (Shaheen and Chan, 2016). This study, therefore, attempts to propose a new dimension of privacy concerns, privacy concerns for traceable information (PCTI) in an e-scooter sharing context, given the fact that e-scooter users may face the risk of not only 
misuse, leakage, and loss of personal information, such as email information, billing addresses, and credit card numbers, but also the unintentional exposure of their location tracking data generated by riding trajectories from the global positioning system (GPS) and tracking sensors embedded in e-scooters, an essential function equipped in most MaaS platforms to track and relocate serviced vehicles. That is, if a rider's name, address, and photo image, along with her/his location information happened to be used for undesired or even criminal purposes, s/he can be tracked to her/his home or exact location at any specific time, even in complicated metropolitan areas. It is proven that if someone with basic coding skills has access to the database, s/he can connect a trip path to an individual without much difficulty (Nelson, 2019). Therefore, the danger of many types of malicious use of traceable location information, such as harassment and stalking, can become a reality. To worsen the situation, it is alleged that major e-scooter sharing companies, such as Bird, Spin, and Lime have already collected a large amount of extra personal information for their commercial use purposes, and the U.S. government has started to use this private information from e-scooter sharing platforms to surveil citizens and target extreme activists or illegal immigrants (Satola, 2018).

As a result, many individuals start to take actions and try to control and protect their privacy, which can be referred to as information privacy protective responses (IPPR) (Son and Kim, 2008). We therefore try to investigate how PCTI affect IPPR. To be more specific, our research purpose is to identify and introduce a new dimension of privacy concerns (i.e., PCTI) and, based on the Antecedent-Privacy ConcernOutcome (APCO) macro model, further examine what factors may cause PCTI and how PCTI can possibly influence IPPR in the context of e-scooter sharing platforms.

The key theoretical contribution of this study is that the concept and measurement of PCTI are firstly proposed and a detailed examination of this concept in terms of privacy concerns for both personal and location information is also conducted. Moreover, drawing on the APCO model, the relationships among several key antecedents for privacy concerns, PCTI, and IPPR are analyzed in the context of e-scooter sharing platforms. For practitioners, the result of this study may benefit many stakeholders of e-scooter sharing platforms, such as platform managers, riders, and even city transportation policy makers.

\section{Literature review}

\subsection{Privacy concerns for traceable information (PCTI)}

Despite the fact that privacy concerns have been examined in various contexts, it is believed that users' privacy concerns involved in the sharing economy are more likely to exceed those in the e-commerce or social media contexts (Young and Quan-Haase, 2013), as more detailed disclosure of personal information (e.g., location and financial information such as GPS coordinate information and credit card numbers) is often a precondition for using the sharing economy services (e.g., UBER) and users cannot be opt-out from disclosing those information, while the website of ecommerce or social media services requires limited personal information (e.g., full name, email, mailing address, etc.) to either browse products or start to use the services (Schoenbachler and Gordon, 2002).

However, extant research in the context of the sharing economy has treated privacy concerns for the sharing economy services similar to those for ecommerce or social media services. For example, Lutz et al. (2017) argued that sharing service users concern about their information privacy as companies may use personal data without their consent or suffer from information breach, which is similar to privacy concerns of other contexts such as e-commerce or social media services. However, in the context of the sharing economy services, especially those enabled by location-based services such as ride-sharing services, we should also consider privacy concerns related to users' traceable trip data from GPS-equipped devices (Hung et al., 2015). In order to locate the 'dock-less' sharing vehicles (i.e., e-scooters) precisely and to propose location-based promotions and ads to (especially frequent) users, service providers tend to store users' trip data at the very granular level and use the data to analyze and predict users' temporal and movement regularity or irregularity (Petersen, 2019). This personal trip data at very granular level at the hand of service providers could be a serious privacy concern for service users. However, users' privacy concerns regarding the traceable information on escooter sharing platforms have not been examined despite its practical urgency and academic attraction. This study therefore attempts to identify and introduce PCTI as a new dimension of privacy concerns on top of other pre-existing concerns, based on the unique characteristics of e-scooter sharing services with traceable users' trip data, which entails users' temporal and movement regularity or irregularity.

\subsection{APCO macro model}




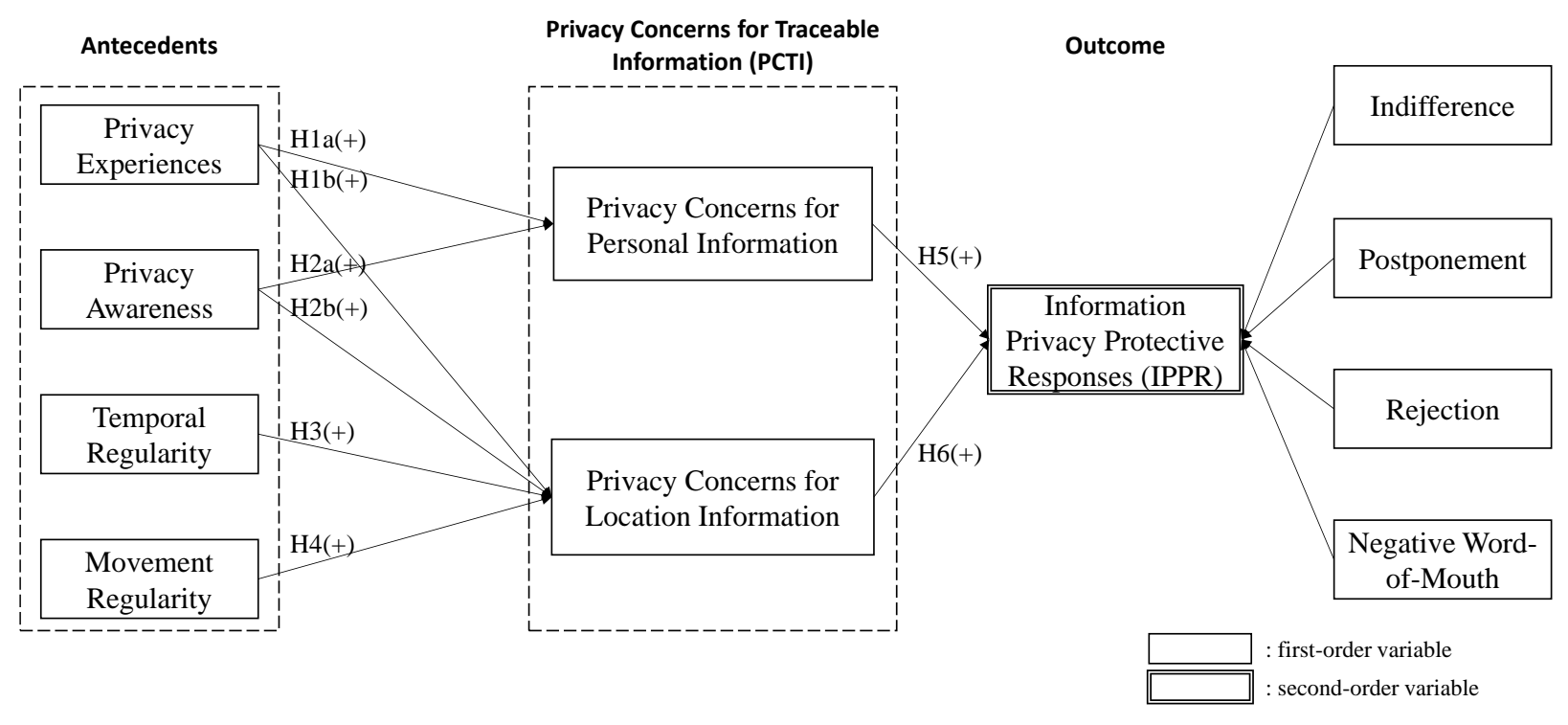

Figure 1. Research model

The Antecedent-Privacy Concern-Outcome (APCO) macro model is a theoretical framework that illustrates relationships among privacy concerns, key affective, cognitive, and personal antecedents, and the outcome of privacy concerns, based on various disciplines, such as IS, organizational behavior, and marketing (Smith et al., 2011). It has been used to investigate phenomena on privacy concerns in the contexts of social media platforms (e.g., Facebook) and location-based services in general (e.g., Lankton and Tripp, 2013; Raschke et al., 2014). To the best of our knowledge, the APCO macro model has not been used as a theoretical framework for privacy concerns related to e-scooter sharing platforms.

The APCO macro model has five antecedents: privacy experiences, privacy awareness, personality differences, demographic differences, and culture (climate). However, Smith et al. (2011) further noted that the original model does not and cannot provide an exhaustive set of antecedents, and various constructs should be included for different contexts. We therefore develop an APCO macro model for e-scooters by replacing three antecedents from the original model (i.e., personality differences, demographic differences, and culture or climate) with two variables representing the unique features of e-scooter sharing services, namely temporal regularity and movement regularity, which demonstrate the riding frequency and riding route predictability, respectively, as discussed in the previous section. Demographic differences are used as control variables in this study and culture- or climaterelated factors are excluded as they are not the major interest of this study as the scope of this study is to investigate privacy concerns of e-scooter users in a single cultural boundary. Moreover, personality differences are not considered in this study because we believe that personality has relatively less influence on PCTI than the two proposed characteristics of e-scooter sharing services (i.e., temporal and movement regularities). However, we admit that a future study should compare the relative impacts of extant antecedents in Smith et al. (2011) and the two proposed antecedents on privacy concerns in the context of e-scooters sharing services.

Privacy concerns, a major construct of this study, has been defined as users' perceptions of what will happen to their online information, and their worries of organizational information privacy practices (Dinev and Hart, 2006; Smith et al., 1996). Users who have higher concerns about their privacy are more likely to feel that their privacy has been threated, either in direct or subtler ways, and respond to it (Son and Kim, 2008). We therefore use information privacy protective responses (IPPR) to measure e-scooter riders' behavioral reactions to privacy concerns.

\subsection{Information privacy protective responses (IPPR)}

Users of e-scooter sharing services could be dissatisfied with how the companies deal with their private information, grow resistance to the services, and want to protect their privacy due to potential risks caused by the traceability of the service. Extant studies on resistance have categorized the concept of 'resistance' into apathy, passive resistance, active resistance, and aggressive resistance (Coetsee, 1999), further narrowed it down to IT resistance, and specified 
the IT resistance into four dimensions, namely, unaware, disinterested, postpone, and reject (Joseph, 2010).

Even though IPPR has been examined in other online environments such as the Internet use or online social interaction (Jiang et al., 2013; Son and Kim, 2008), little effort has been made to investigate IPPR when individuals have to disclose their personal information to use e-scooters through the services provided on smartphone applications. Accordingly, how users respond to protect their privacy, other than the direct refusal, is one of the main research purposes of this study. We therefore develop four categories of IPPR based on extant studies on IT resistance (Joseph, 2010), namely, indifference, postponement, rejection, and negative word-of-mouth in the context of e-scooter sharing platforms. These categories are slightly different from Joseph (2010)'s category. We do not think that e-scooter users will be 'unaware' of the services because they voluntarily become a user and disclose personal information, but they could be 'indifferent' to information privacy (similar to 'disinterested' in Joseph (2010)'s list). We keep postponement and rejection as they are from Joseph (2010)'s list and added negative word-of-mouth because this is one of tangible responses to information privacy concerns used in any online or mobile platforms that allow users to express their opinions about the service.

\section{Research model and hypotheses}

As shown in Figure 1, drawing on the APCO macro model, eight hypotheses were developed. PCTI, including privacy concerns for both personal and location information mediates the relationships between four antecedents and IPPR. IPPR is a secondorder variable that includes four formative first-order variables from indifference (i.e., the most passive resistance) to postponement, rejection, and even to negative word-of-mouth (i.e., the most aggressive resistance).

\subsection{The relationships between antecedents and PCTI}

As to privacy experiences, it has been empirically validated by Smith et al. (1996) that consumers who have any experiences related to privacy leakage or breach are more likely to show higher concerns towards their information provided to the companies. The positive relationship between privacy experiences and privacy concerns was further verified in a locationbased service environment ( $\mathrm{Xu}$ and Gupta, 2012). We therefore argue that if an e-scooter user has bad experiences regarding privacy leakage and breach issues before, privacy concerns for both personal information and location information will increase. We thus hypothesize:

Hla: Privacy experiences have a positive impact on privacy concerns for personal information.

H1b: Privacy experiences have a positive impact on privacy concerns for location information.

Privacy awareness refers to how users are aware of corporative privacy practices (Malhotra et al., 2004; Phelps et al., 2000). Cespedes and Smith (1993) found that consumers' information privacy concerns increase if they are aware that companies share their private information with unauthorized third parties or collect information without their consent. We believe that this finding also holds for both privacy concerns for personal and location information in the context of escooter sharing services. When an e-scooter user finds that the service provider shared her/his personal identifiable information and traceable location information or has the terms of agreement including users' unintended consent that the company is allowed to share private information with third parties without further notification with users, s/he will have more concerns for both personal information and location information. We thus hypothesize:

H2b: Privacy awareness has a positive impact on privacy concerns for personal information.

H2b: Privacy awareness has a positive impact on privacy concerns for location information.

Temporal regularity and movement regularity show the frequency in riding shared e-scooters and the predictability of riding routes, respectively (Lee et al., 2011; Zhong et al., 2016). To be more specific, high temporal regularity implies that a rider uses shared escooters frequently in a regular basis, while high movement regularity indicates that an individual rides e-scooters to visit only limited number of locations, for example, by commuting between home and workplace.

For temporal regularity, we propose that if riders use shared e-scooters more frequently, their privacy concerns for location information would increase as a large quantity of location data has been generated on escooter sharing programs. As to movement regularity, if the users' riding routes are fairly fixed (i.e., high movement regularity), their locations in certain time of a day would become easy to be predicted, and if their personal information got exposed, they can be tracked to an exact location at a specific time, which raises privacy concerns for their location data to a high level. 
Table 1. Operational definitions of constructs

\begin{tabular}{|c|c|c|c|}
\hline Construct & & Operational Definition & Reference \\
\hline \multicolumn{4}{|c|}{ Independent Variables } \\
\hline $\begin{array}{l}\text { Privacy } \\
\text { Experiences }\end{array}$ & \multicolumn{2}{|c|}{$\begin{array}{l}\text { The degree of a user's personal information has been abused or } \\
\text { attacked before. }\end{array}$} & Smith et al. (1996) \\
\hline $\begin{array}{l}\text { Privacy } \\
\text { Awareness }\end{array}$ & \multicolumn{2}{|c|}{$\begin{array}{l}\text { The degree to which a user is aware of privacy practices of e- } \\
\text { scooter sharing platforms. }\end{array}$} & $\begin{array}{l}\text { Malhotra et al. (2004); } \\
\text { Phelps et al. (2000) }\end{array}$ \\
\hline $\begin{array}{l}\text { Temporal } \\
\text { Regularity }\end{array}$ & \multicolumn{2}{|c|}{ The extent to how frequently a user uses e-scooter sharing services } & Zhong et al. (2016) \\
\hline $\begin{array}{l}\text { Movement } \\
\text { Regularity }\end{array}$ & \multicolumn{2}{|c|}{ The extent to how predictable a user's riding route is } & Lee et al. (2011) \\
\hline \multicolumn{4}{|c|}{ Mediating Variables } \\
\hline $\begin{array}{l}\text { Privacy } \\
\text { Concerns for } \\
\text { Personal } \\
\text { Information }\end{array}$ & \multicolumn{2}{|c|}{$\begin{array}{l}\text { The degree to which a user perceives that } \mathrm{s} / \text { he concerns about the } \\
\text { possible loss of privacy as a result of personal information } \\
\text { disclosure to e-scoter sharing platforms }\end{array}$} & Xu et al. (2009) \\
\hline $\begin{array}{l}\text { Privacy } \\
\text { Concerns for } \\
\text { Location } \\
\text { Information }\end{array}$ & \multicolumn{2}{|c|}{$\begin{array}{l}\text { The degree to which a user perceives that s/he concerns about the } \\
\text { possible loss of privacy as a result of location information } \\
\text { disclosure to e-scoter sharing platforms }\end{array}$} & Xu et al. (2009) \\
\hline \multicolumn{4}{|c|}{ Dependent Variable } \\
\hline \multirow{4}{*}{$\begin{array}{l}\text { Information } \\
\text { Privacy } \\
\text { Protective } \\
\text { Responses } \\
\text { (IPPR) }\end{array}$} & Indifference & $\begin{array}{l}\text { The degree to which a user avoids receiving } \\
\text { information of e-scooter sharing services }\end{array}$ & Coetsee (1999) \\
\hline & Postponement & $\begin{array}{l}\text { The degree to which a user delays using e- } \\
\text { scooter sharing services }\end{array}$ & $\begin{array}{l}\text { Szmigin and Foxall } \\
(1998)\end{array}$ \\
\hline & Rejection & $\begin{array}{l}\text { The degree to which a user has no intention to } \\
\text { use e-scooter sharing services. }\end{array}$ & Mittelstaedt et al. (1976) \\
\hline & $\begin{array}{l}\text { Negative word- } \\
\text { of-mouth }\end{array}$ & $\begin{array}{l}\text { The degree to which a user shares negative } \\
\text { experiences with others about e-scooter sharing } \\
\text { services. }\end{array}$ & Son and Kim (2008) \\
\hline
\end{tabular}

However, we argue that both temporal regularity and movement regularity will not affect privacy concerns for personal information, as it does not vary depending on use frequency and riding routes predictability, while location information can change dramatically with temporal and movement regularities. Our hypotheses, then, are as follows:

H3: Temporal regularity has a positive impact on privacy concerns for location information.

H4: Movement regularity has a positive impact on privacy concerns for location information.

\subsection{The relationships between PCTI and IPPR}

Son and Kim (2008) found a positive relationship between privacy concerns and IPPR, as user dissatisfaction towards the companies grows when private information got mishandled. In the context of escooter sharing services, the services are associated not only with private concerns for personal information, but also with those for location information automatically generated from the GPS function. We therefore propose both types of privacy concerns can trigger (are positively related to) users' IPPR. Accordingly, we hypothesize:

H5: Privacy concerns for personal information have a positive impact on IPPR.

H6: Privacy concerns for location information have a positive impact on IPPR.

\section{Research methodology}

In order to investigate proposed hypotheses, we plan to collect survey data from our target population, those who have experience in using major e-scooter sharing services in the U.S., including Bird, Spin, and Lime. While the nature of and technologies behind those different e-scooter sharing services are similar to one another, it is possible that each service has different characteristics with regard to their service offerings, data collection, and privacy policies, and those specific characteristics could be related to the 
key variables of this study. Therefore, when we develop items for survey questionnaire and administer survey data collection, we will be mindful about the specificity of each one of the services and make sure that the specificity of different services does not affect general tendency of users with regard to the latent variables of our research model. Data analysis will be done using a correlation-based structural equation modeling technique.

Table 1 demonstrates operational definitions of constructs. The measurement items will be adapted from extant studies and the validity and reliability of the measurement items will be tested. The measurement scales for variables will be presented in the conference. We will employ a seven-point Likert scale (i.e., strongly disagree - strongly agree or never to always, etc.) to examine how e-scooter sharing platform users in the U.S. perceive about the services in terms of our research constructs and items. Some demographic information such as gender, age group, income and education level, and job categories, etc. will be collected and tested as control variables.

\section{Expected implications}

This study is expected to contribute to the literature on information privacy by providing empirical evidence on the relationships among proposed antecedents, two dimensions of privacy concerns, and IPPR in the context of e-scooter services. More specifically, PCTI is identified and further investigated as a form of privacy concerns related to the use of Mobility-as-a-Service (Maas) in general and e-scooters in particular and this dimension of privacy concerns is added to the extant dimension of privacy concerns (for personal information). Further, to the best of our knowledge, this study is one of the first attempts to extend the APCO macro model with new IPPR as behavioral reactions, including indifference, postponement, rejection, and negative word-of-mouth to better fit the context of the e-scooter sharing service.

Our research results will have practical implications that are beneficial to various stakeholders in the escooter sharing context. For e-scooter sharing program managers, how users react to protect their privacy can be better understood; even though a direct rejection is out of options, users will employ other passive or active resistant reactions. Managers are also encouraged to alleviate privacy concerns of users by improving their privacy protection in two proposed dimensions. Finally, understanding users' information privacy protective responses can reversely contribute to boosting individuals' continuous use of e-scooter sharing services. If users' privacy concerns are mitigated, their behavior responses are more likely to convert from resistance to continuous use, which will help maintain more existing users and expand the market share of e-scooter sharing companies.

\section{Acknowledgements}

This work was supported by the Ministry of Education of the Republic of Korea and the National Research Foundation of Korea (NRF2017S1A5B8059804).

\section{References}

[1] Cespedes, F. V., and Smith, H. J., "Database Marketing: New Rules for Policy and Practice", Sloan Management Review, Vol. 34, No. 4, 1993, pp. 7-27.

[2] Chang, Y., Wong, S. F., Libaque-Saenz, C. F., and Lee, H., "The Role of Privacy Policy on Consumers' Perceived Privacy”, Government Information Quarterly, Vol. 35, No. 3, 2018, pp. 445-459.

[3] Coetsee, L., "From Resistance to Commitment", Public Administration Quarterly, 1999, pp. 204-222.

[4] Dickey, M. R., "Shared Electric Scooter Rides Accounted for $45.8 \%$ of All Micromobility Trips in 2018", 2019, Available at https://techcrunch.com/2019/04/17/shared-electric-scooterrides-accounted-for-45-8-percent-of-all-micromobility-tripsin-2018/.

[5] Dinev, T., and Hart, P., "An Extended Privacy Calculus Model for E-Commerce Transactions", Information Systems Research, Vol. 17, No. 1, 2006, pp. 61-80.

[6] Gindrat, R., "Mobility as a Service Models Begin to Emerge", 2018, Available at:

https://medium.com/bestmile/mobility-as-a-service-modelsbegin-to-emerge-

47a967d7cf3c?sk=ca0345a15a01c6405ea1f6cf9d5d25c6.

[7] Hamari, J., Sjöklint, M., and Ukkonen, A., "The Sharing Economy: Why People Participate in Collaborative Consumption", Journal of the Association for Information Science and Technology, Vol. 67, No. 9, 2016, pp. $2047-$ 2059.

[8] Hung, C. C., Peng, W. C., and Lee, W. C., "Clustering and Aggregating Clues of Trajectories for Mining Trajectory Patterns and Routes", The International Journal on Very Large Data Bases, Vol. 24, No. 2, 2015, pp. 169-192.

[9] Jiang, Z., Heng, C. S., and Choi, B. C., "Privacy Concerns and Privacy-Protective Behavior in Synchronous Online Social Interactions", Information Systems Research, Vol. 24, No. 3, 2013, pp. 579-595. 
[10] Joseph, R. C., "Individual Resistance to It Innovations", Communications of the ACM, Vol. 53, No. 4, 2010, pp. 144-146.

[11] Lankton, N. K., and Tripp, J. F., "A Quantitative and Qualitative Study of Facebook Privacy Using the Antecedent-Privacy Concern-Outcome Macro Model", Proceedings of the 19th Americas Conference on Information Systems (AMCIS) 2013, Chicago, Illinois, U. S. A., 2013, pp. $1-12$.

[12] Lee, R., Wakamiya, S., and Sumiya, K., "Discovery of Unusual Regional Social Activities Using Geo-Tagged Microblogs", World Wide Web, Vol. 14, No. 4, 2011, pp. 321-349.

[13] Lutz, C., Hoffmann, C. P., Bucher, E., and Fieseler, C., "The Role of Privacy Concerns in the Sharing Economy", Information, Communication \& Society, 2017, pp. 1-21.

[14] Malhotra, N. K., Kim, S. S., and Agarwal, J., "Internet Users' Information Privacy Concerns (IUIPC): The Construct, the Scale, and a Causal Model", Information Systems Research, Vol. 15, No. 4, 2004, pp. 336-355.

[15] Mittelstaedt, R. A., Grossbart, S. L., Curtis, W. W., and Devere, S. P., "Optimal Stimulation Level and the Adoption Decision Process", Journal of Consumer Research, Vol. 3, No. 2, 1976, pp. 84-94.

[16] Nelson, L. J., "Data Privacy Debate Emerges out of L.A.'s Scooter Craze", 2019, Available at: https://www.govtech.com/transportation/Data-Privacy-

Debate-Emerges-Out-of-LAs-Scooter-Craze.html.

[17] Petersen, A. B., "Scoot over Smart Devices: The Invisible Costs of Rental Scooters", Surveillance \& Society, Vol. 17, No. 1-2, 2019, pp. 191-197.

[18] Phelps, J., Nowak, G., and Ferrell, E., "Privacy Concerns and Consumer Willingness to Provide Personal Information", Journal of Public Policy \& Marketing, Vol. 19, No. 1, 2000, pp. 27-41.

[19] Raschke, R. L., Krishen, A. S., and Kachroo, P., "Understanding the Components of Information Privacy Threats for Location-Based Services", Journal of Information Systems, Vol. 28, No. 1, 2014, pp. 227-242.

[20] Satola, A., "Are Bird Scooters Flying Away with User Data?" 2018, Available at:

https://www.michigandaily.com/section/columns/alexandersatola-bird-scooters-and-data-security.

[21] Schoenbachler, D. D., and Gordon, G. L., "Trust and Customer Willingness to Provide Information in Database-
Driven Relationship Marketing", Journal of Interactive Marketing, Vol. 16, 2002, pp. 2-16.

[22] Shaheen, S., and Chan, N., "Mobility and the Sharing Economy: Potential to Overcome First- and Last-Mile Public Transit Connections", 2016, UC Berkeley: Transportation Sustainability Research Center. Available at https://escholarship.org/uc/item/8042k3d7.

[23] Smith, H. J., Dinev, T., and Xu, H., "Information Privacy Research: An Interdisciplinary Review", MIS Quarterly, Vol. 354, No. 4, 2011, pp. 989-1016.

[24] Smith, H. J., Milberg, S. J., and Burke, S. J., "Information Privacy: Measuring Individuals' Concerns about Organizational Practices", MIS Quarterly, Vol. 20, No. 2, 1996, pp. 167-196.

[25] Son, J. Y., and Kim, S. S., "Internet Users' Information Privacy-Protective Responses: A Taxonomy and a Nomological Model”, MIS Quarterly, Vol. 32, No. 3, 2008, pp. 503-529.

[26] Sun, Y., Wang, N., Shen, X. L., and Zhang, J. X., "Location Information Disclosure in Location-Based Social Network Services: Privacy Calculus, Benefit Structure, and Gender Differences", Computers in Human Behavior, Vol. 52, 2015, pp. 278-292.

[27] Szmigin, I., and Foxall, G., "Three Forms of Innovation Resistance: The Case of Retail Payment Methods", Technovation, Vol. 18, No. 6-7, 1998, pp. 459468.

[28] Xu, H., and Gupta, S., "The Effects of Privacy Concerns and Personal Innovativeness on Potential and Experienced Customers' Adoption of Location-Based Services", Electronic Markets, Vol. 19, No. 2-3, 2012, pp. 137-149.

[29] Xu, H., Teo, H. H., Tan, B. C., and Agarwal, R., “The Role of Push-Pull Technology in Privacy Calculus: The Case of Location-Based Services", Journal of Management Information Systems, Vol. 26, No. 3, 2009, pp. 135-174.

[30] Young, A. L., and Quan-Haase, A., "Privacy Protection Strategies on Facebook: The Internet Privacy Paradox Revisited Information", Communication \& Society, Vol. 16, No. 4, 2013, pp. 479-500.

[31] Zhong, C., Batty, M., Manley, E., Wang, J., Wang, Z., Chen, F., and Schmitt, G., "Variability in Regularity: Mining Temporal Mobility Patterns in London, Singapore and Beijing Using Smart-Card Data", PloS One, Vol. 11, No. 2, 2016, pp. 149-222. 